\title{
Dispensing and practice use patterns, facilitators and barriers for uptake of ulipristal acetate emergency contraception in British Columbia: a mixed-methods study
}

\author{
Michelle C. Chan MD MHSc, Sarah Munro PhD, Laura Schummers ScD, Arianne Albert PhD, \\ Frannie Mackenzie BA, Judith A. Soon RPh PhD, Parkash Ragsdale BSc(Pharm) RPh, \\ Brian Fitzsimmons MD, Regina Renner MD MPH
}

\section{Abstract}

Background: Ulipristal acetate $30 \mathrm{mg}$ became available as prescription-only emergency contraception in British Columbia, Canada, in September 2015, as an addition to over-the-counter levonorgestrel emergency contraception. In this study, we determined dispensing and practice use patterns for ulipristal acetate, as well as facilitators of and barriers to emergency contraception for physicians, pharmacists and patients in BC.

Methods: In the quantitative component of this mixed-methods study, we examined ulipristal acetate use from September 2015 to December 2018 using a database that captures all outpatient prescription dispensations in BC (PharmaNet) and another capturing market sales numbers for all oral emergency contraception in BC (IQVIA). We analyzed the quantitative data descriptively. We conducted semistructured interviews from August to November 2019, exploring barriers and facilitators affecting the use of ulipristal acetate. We performed iterative qualitative data collection and thematic analysis guided by Michie's Theoretical Domains Framework.

Results: Over the 3-year study period, 318 patients filled 368 prescriptions for ulipristal acetate. Use of this agent increased between 2015 and 2018. However, levonorgestrel use by sales (range 118 897-129 478 units/yr) was substantially higher than use of ulipristal acetate (range 128-389 units/yr). In the 39 interviews we conducted, from the perspectives of 12 patients, 12 community pharmacists, and 15 prescribers, we identified the following themes and respective theoretical domains as barriers to access: low awareness of ulipristal acetate (knowledge), beliefs and experiences related to shame and stigma (beliefs about consequences), and multiple health system barriers (reinforcement).

Interpretation: Use of ulipristal acetate in BC was low compared with use of levonorgestrel emergency contraception; lack of knowledge, beliefs about consequences and health system barriers may be important impediments to expanding use of ulipristal acetate. These findings illuminate potential factors to explain low use of this agent and point to the need for additional strategies to support implementation.

U lipristal acetate $30 \mathrm{mg}$ became available as prescription-only oral emergency contraception in British Columbia, Canada, in September 2015, as an addition to over-the-counter levonorgestrel emergency contraception. Currently in Canada, recommended emergency contraception options include oral methods (ulipristal acetate or levonorgestrel) or intrauterine device (copper). ${ }^{1}$ Oral emergency contraception prevents pregnancy by delaying ovulation after intercourse without contraception or intercourse with contraceptive failure. ${ }^{2,3}$

Ulipristal acetate is superior to levonorgestrel emergency contraception in preventing pregnancy. ${ }^{4}$ It is more effective up to 120 hours from intercourse, compared with 72 hours for levonorgestrel, and it is more effective in those who are overweight. ${ }^{1,5}$ Accordingly, current Canadian practice guidelines recommend ulipristal acetate for emergency contraception in people with self-reported body mass index (BMI) above 25, which includes about $45 \%$ of reproductive-aged (18-34 yr) women in Canada. ${ }^{1,6}$

Canadian research on emergency contraception has explored patients' barriers to accessing levonorgestrel and counselling initiatives. ${ }^{7-11}$ Most studies have been quantitative and involved pharmacists only, patients only or prescribers only. ${ }^{12-26}$ Prior studies have often included a proxy approach,

\section{Competing interests: None declared.}

This article has been peer reviewed.

Correspondence to: Michelle Chan, michelle.chan1@cw.bc.ca CMAJ Open 2021 November 30. DOI:10.9778/cmajo.20200193 
such as the use of secret shoppers, who are not truly representative of patients; we therefore aimed to include self-identified patient voices. ${ }^{16-18}$

In this study, we described dispensing patterns for ulipristal acetate and compared them with dispensing patterns for levonorgestrel oral therapy, both primarily indicated for emergency contraception in BC. We also explored facilitators of and barriers to use of ulipristal acetate with prescribers, pharmacists and patients, the key stakeholders involved in the prescription and medication process in BC.

\section{Methods}

\section{Design and setting}

We conducted this mixed-methods study with concurrent quantitative and qualitative approaches. ${ }^{27} \mathrm{We}$ used quantitative analysis to describe the distribution of use of emergency contraception types within BC. Given the lack of Canadian studies investigating ulipristal acetate use since it was first marketed in Canada, we chose interviews for this study to provide richer exploratory data. The qualitative analysis explored reasons for the trends in use, as well as barriers and facilitators, and helped us to interpret our quantitative findings. We analyzed our findings using a contiguous approach to allow expansion or confirmation of the respective findings. ${ }^{27}$

The study was conducted in BC, which has provincially organized health care delivery. In 2018, 35.8\% $(n=174300)$ of BC women aged 18-34 years self-reported being overweight or obese. ${ }^{6}$

\section{Participants}

Two of the authors (M.C.C. and F.M.) recruited a purposeful sample of urban and rural participants from BC for one-onone semistructured interviews about knowledge, beliefs and experiences with emergency contraception. We recruited patients, prescribers and pharmacists, half urban and half rural in each group, to represent the province. Patient inclusion criteria were the following: female (including cis and transgender individuals who had a uterus, vagina and ovaries), age 18-45 years, English-speaking, requesting reproductive health care in BC, sexually active and trying to prevent a pregnancy sometime between 2015 and the interview date, at risk of getting pregnant (e.g., not having a permanent method of sterilization, such as having their "tubes tied" or hysterectomy, with removal of the uterus, or having male sexual partners who did not have a permanent method of sterilization, specifically "vasectomy"), and having access to email and telephone. Exclusion criteria for patients were inability to consent and not at risk of pregnancy.

Inclusion criteria for prescribers (physicians and nurse practitioners), community pharmacists and nurses were as follows: caring for reproductive-aged women (18-45 yr), practising in BC, English-speaking and having access to email and telephone. To explore potential barriers, we included nurses certified in contraceptive care, who can provide contraception but cannot prescribe. We defined urban regions by Statistics Canada census metropolitan areas and all other regions as rural. ${ }^{28}$

\section{Data sources}

\section{Quantitative data}

We measured use of oral ulipristal acetate and levonorgestrel emergency contraception by combining data for provincial prescription dispensations and pharmaceutical sales.

To capture utilization of ulipristal acetate since it became available, we used PharmaNet to identify all prescriptions from September 2015 to December 2018, the most recent data available at the time of our data request. PharmaNet is a province-wide prescription database,${ }^{29}$ managed by the $\mathrm{BC}$ Ministry of Health, that links all pharmacies; it allows health care providers to access up-to-date outpatient prescription records for each patient. Although the validity and reliability of PharmaNet for ulipristal acetate prescriptions have not been examined, it is the most comprehensive prescription database in BC and the largest universal prescription database in Canada. ${ }^{30}$

Levonorgestrel is available over the counter, so we could not use prescriptions to measure its use. Instead, we obtained from IQVIA the number of ulipristal acetate and levonorgestrel emergency contraception wholesale units sold in $\mathrm{BC}$ during the study period. IQVIA is a privately held market research and consulting firm serving the Canadian health care market, with their own auditing processes. ${ }^{31}$

\section{Qualitative data}

M.C.C. and F.M. recruited participants province-wide using gender, sexuality and person-first materials. ${ }^{32}$ F.M., M.C.C. and R.R. developed a social media toolkit that was shared with collaborating provincial organizations for their use (Appendix 1, part A, available at www.cmajopen.ca/content/9/4/E1097/ suppl/DC1). We recruited patients through provincial sexual health clinics and social media networks, pharmacists by province-wide faxing to community pharmacies and prescribers through provincial practice e-networks. Recruitment materials were linked to an intake form (REDCap survey software) that was used to confirm that potential participants met the inclusion criteria. The intake form also gathered data on patient age, BMI (based on height and weight) and emergency contraceptive use.

Recruitment started in July 2019 and continued until purposeful sampling was complete. ${ }^{3-36}$ Previous reviews have noted thematic saturation with purposeful sampling within 12 interviews. ${ }^{37}$ Therefore, the sample size goal was 12 patients, 12 pharmacists and 12 prescribers. Nurses are certified in contraceptive care to provide contraception but cannot prescribe within the province of $\mathrm{BC}$; therefore, we included an additional 3 nurses and interviewed them with the prescriber group in an attempt to capture their unique role in relation to emergency contraception.

We conducted interviews until we achieved saturation in our data collection, sampling and analysis. We also sought to recruit participants until our data sufficiently represented a range of the pre-identified factors from our purposeful sampling strategies, ${ }^{38}$ as described above. There were no preexisting relationships with study participants, we did not 
track nonparticipation, and there was no participant checking (e.g., we did not return transcripts to or share study findings with the participants).

We adapted the interview guide from Hussainy's protocol for ACCESS ${ }^{36}$ using Michie's Theoretical Domains Framework $;{ }^{39}$ see Appendix 1, part B, for 2 interview guides, one for patients and the other for prescribers and pharmacists. The framework explores implementation problems and informs potential interventions to enhance access. ${ }^{36}$ It includes domains such as knowledge (awareness of something) and skills (abilities or proficiencies acquired through practice). ${ }^{36}$ M.C.C. adapted and S.M. reviewed the interview guides to include patient- and prescriber-specific questions in each domain. ${ }^{33,36}$ Although the original protocol was designed only for interviewing patients and pharmacists, we subsequently added questions for prescribers within applicable domains. For example, we asked prescribers about their prescribing experiences, whereas we asked patients about their experiences in accessing oral emergency contraceptives.

All coauthors had the opportunity to review the guides before study commencement. Although the guides were not piloted, expert review was performed by physicians, pharmacists and S.M. (an expert in patient engagement, plain language materials and qualitative interviewing, who provides regular detailed review of patient-facing materials for clinicians and researchers). M.C.C. also made modifications and additions to interview guides iteratively as needed. We conducted interviews from August 2019 until the sample size was complete (in November 2019).

M.C.C., a cis-female of colour who is a feminist obstetriciangynecologist, conducted all interviews and obtained verbal consent for participation in the study. This author has completed a Master's-level course about qualitative research and has conducted telephone surveys and research interviews in other studies. We recorded the interviews on a secure virtual conferencing platform. Each participant received a $\$ 50$ gift card to acknowledge their contribution to the study.

\section{Statistical analysis}

\section{Quantitative data}

Following the plan developed by M.C.C., L.S., A.A., J.A.S. and R.R., the descriptive analysis included tabulating the number of ulipristal acetate prescriptions dispensed, as well as the numbers of ulipristal acetate and levonorgestrel products sold to pharmacies each year. We summarized patients' median age and the proportion of prescribers in each practice type and specialty for all dispensed prescriptions of ulipristal acetate. A.A. conducted all of the quantitative analyses using $\mathrm{R}$ software, version 3.5.3.

\section{Qualitative data}

We engaged a professional transcription service to transcribe all audio recordings. M.C.C. and F.M. cleaned the transcripts to ensure de-identification and checked for quality and accuracy. M.C.C. led a hybrid inductive-deductive thematic analysis informed by a critical feminist reproductive justice lens. ${ }^{40,41}$ M.C.C. read and reread the transcripts to gain familiarity with the data. M.C.C. and S.M. generated a preliminary inductive codebook, and then deductively matched the codes to the theoretical domains (themes). M.C.C. coded a transcript from each participant group (patients, prescribers, pharmacists) to test the preliminary codebook for fit and relevance and then reviewed the coding with S.M. They (M.C.C. and S.M.) made minor revisions to the codebook for conceptual fit. M.C.C. then applied the same codebook to patient, pharmacist and prescriber transcripts with the assistance of NVivo 12 Pro software. Throughout the analysis of qualitative data, we engaged in verification strategies, including bracketing exercises, maintaining an audit trail and field notes (M.C.C.), and frequent team discussions of analysis in progress.

\section{Mixed-methods integration}

The analysis was a simultaneous qualitative and quantitative approach, whereby the qualitative analysis explored reasons for trends in use and helped in interpreting, explaining and providing context for our quantitative findings, and vice versa. We provided all team members with the analysis in progress and the final results, and used a mixed-methods data matrix to compare, contrast and interpret the results. Please see Appendix 1, part C, for details.

\section{Ethics approval}

This study received ethics approval from the University of British Columbia Children's and Women's Research Ethics Board (H18-03350).

\section{Results}

Our analyses of BC Pharmanet data showed that during the 3 -year period, 318 patients filled a total of 368 prescriptions for ulipristal acetate: 281 filled 1 prescription, 26 filled 2 prescriptions, and 11 filled 3 or more prescriptions. The mean age of users of ulipristal acetate was 29.8 (standard deviation 7.7) years; $25 \%$ were younger than 24 years, whereas $25 \%$ were older than 35. The prescribers consisted of 354 physicians, of whom 18 $(5 \%)$ were obstetrics and gynecology specialists and $9(3 \%)$ were nurse practitioners. Table 1 shows that the number of ulipristal acetate packages purchased by pharmacies and dispensed to

\begin{tabular}{|c|c|c|c|}
\hline \multirow[b]{2}{*}{ Year } & \multirow{2}{*}{$\begin{array}{l}\text { No. of UPA } \\
\text { prescriptions } \\
\text { dispensed* }^{*}\end{array}$} & \multicolumn{2}{|c|}{ Drug; total no. of units sold† } \\
\hline & & Levonorgestrel & UPA \\
\hline $2015 \ddagger$ & $<5$ & 56021 & 44 \\
\hline 2016 & 48 & 129478 & 128 \\
\hline 2017 & 149 & 118897 & 195 \\
\hline 2018 & 167 & 124910 & 389 \\
\hline \multicolumn{4}{|c|}{$\begin{array}{l}\text { Note: UPA = ulipristal acetate. } \\
{ }^{*} \text { Data from provincial prescription database (PharmNet). } \\
\text { †Data from commercial sales database (IQVIA). } \\
\text { †Data for } 2015 \text { represent September to December, not the full calendar year. }\end{array}$} \\
\hline
\end{tabular}


patients increased over time. According to sales data, use of levonorgestrel (range 118 897-129 478 units/yr) was substantially higher than use of ulipristal acetate (range 128-389 units/yr).

We interviewed 12 patients, 12 pharmacists, 12 prescribers and 3 nurses (Table 2). The interviews lasted 1-1.5 hours. Patients' median age was 27 years, and half had a BMI over 25. All pharmacists and prescribers reported that they had clinical experience with levonorgestrel, but only a third of the pharmacists and prescribers had any clinical experience with ulipristal acetate.

The qualitative analysis reached saturation and identified more barriers than facilitators to use of emergency contraception. There was low awareness of ulipristal acetate, and participants were only able to provide information about their experiences primarily with levonorgestrel oral emergency contraception. Given such low awareness of ulipristal acetate, we could not compare barriers and facilitators for ulipristal acetate and levonorgestrel separately.

\begin{tabular}{|c|c|}
\hline Characteristic & No. of participants* \\
\hline \multicolumn{2}{|l|}{ Patients $(n=12)$} \\
\hline Age, yr, median (range) & $27(21-39)$ \\
\hline BMI, median (IQR) & $24.60(21.66-30.76)$ \\
\hline \multicolumn{2}{|l|}{ Location } \\
\hline Rural & 6 \\
\hline Urban & 6 \\
\hline \multicolumn{2}{|l|}{ Emergency contraception experience } \\
\hline Reported previous attempts to access & 9 \\
\hline Reported previous use & 10 \\
\hline \multicolumn{2}{|l|}{ Pharmacists $(n=12)$} \\
\hline \multicolumn{2}{|l|}{ Location } \\
\hline Rural & 6 \\
\hline Urban & 6 \\
\hline \multicolumn{2}{|l|}{ Dispensing experience } \\
\hline Levonorgestrel & 12 \\
\hline Ulipristal acetate & 5 \\
\hline \multicolumn{2}{|l|}{ Prescribers $(n=15)$} \\
\hline \multicolumn{2}{|l|}{ Type } \\
\hline Nurse† & 3 \\
\hline Physician & 12 \\
\hline \multicolumn{2}{|l|}{ Location } \\
\hline Rural & 8 \\
\hline Urban & 7 \\
\hline \multicolumn{2}{|l|}{ Prescribing experience } \\
\hline Levonorgestrel & 15 \\
\hline Ulipristal acetate & 4 \\
\hline \multicolumn{2}{|c|}{$\begin{array}{l}\text { Note: } \mathrm{BMI}=\text { body mass index, IQR = interquartile range. } \\
\text { *Unless otherwise specified. } \\
\text { †Nurse practitioner or nurse with certification in contraception. }\end{array}$} \\
\hline
\end{tabular}

We identified 3 core themes mapped to the Theoretical Domains Framework: knowledge, beliefs about consequences and reinforcement. Table 3 presents representative quotations for each theme.

\section{Theme 1: Low awareness about ulipristal acetate (knowledge)}

The knowledge domain defines the awareness of the existence of something. Patients were aware of levonorgestrel and the availability of this product at community pharmacies. However, many study participants were unaware of ulipristal acetate or the requirement for a prescription. Consequently, the absence of awareness related to ulipristal acetate resulted in barriers to accessing this drug. Patients were aware of the potential for emergency contraception to prevent pregnancy after intercourse. Patients showed limited understanding of how emergency contraception works, and some feared that the medication could be harmful or dangerous.

When asked about awareness and knowledge of emergency contraception, patients perceived a dearth of comprehensive sexual education contributing to low reproductive health literacy.

Prescribers and pharmacists acquired knowledge about emergency contraception during didactic professional training programs, self-directed continuing education courses and exposure to the products in clinical practice. The prescribers and pharmacists reported challenges in learning about newly available products, such as ulipristal acetate, unless motivated by a clinical encounter.

Prescribers referenced, in general terms, the clinical practice guidelines of the Society of Obstetricians and Gynaecologists of Canada as the standard of care. However, they described barriers to knowledge acquisition, such as memberonly access to the society's resources.

Lack of knowledge and the challenge to be aware of new emergency contraception options in all groups compounded each other and were associated with low use of ulipristal acetate. The patients did not know to ask for ulipristal acetate, and the prescribers did not know to recommend it.

\section{Theme 2: Beliefs about or experience of shame and stigma (beliefs about consequences)}

Our analysis of interviews indicated that shame and stigma were barriers to access. This result mapped to the theoretical domain of beliefs about consequences, defined as the acceptance of the truth, reality or validity about an ability, talent or facility that a person can put to constructive use.

Patients shared beliefs and feelings of shame surrounding potential reasons for needing emergency contraception and accessing it.

Prescribers and pharmacists shared opinions about acceptable use of emergency contraception. For example, some prescribers and pharmacists believed that for persons not already using a regular or reliable form of contraception, attempts to access emergency contraception represented perceived irresponsibility, rather than an "emergency" situation. Similarly, some prescribers and pharmacists felt that accessing emergency contraception multiple times as a form of family planning was not acceptable. 
Table 3: Qualitative results: selected quotes

Theme

Low awareness concerning

UPA (knowledge)

Beliefs about or experience of shame and stigma (beliefs about consequences)

Health care system barriers (reinforcement)
Selected quotes

\section{Patients}

My understanding is if you were to have unprotected sex or ... reason why you would think that you might get pregnant, the next day you would take the pill. I understand that it hurts your body a little bit. You get quite sick ... And, yeah, that nobody really wants to take it because it kind of makes you a little bit sick. (R63, patient)

I think everybody should have sex education and I know not everybody in $\mathrm{BC}$ has access to ... sexual education ... But I think that any time somebody's talking about contraceptives or sex or sexual health or sexuality to youth in schools, I think there needs to be a conversation that's age appropriate, when they start talking about contraception to be talking about emergency contraception as well. (R17, patient)

\section{Prescribers and pharmacists}

Just the need to know about what's up to date and particularly like I said, because I don't recall recently having anybody come in and actually asking me for it. (R40, prescriber)

I mean, a lot of societies are like that ... previously we could read the whole guideline and then we were limited to just reading the highlights and then recently we were just not allowed. (R56, prescriber)

\section{Patients}

Because I really feel that there's a sense of you only take this if you really screwed up this time. (R30, patient)

I think when it comes to having something that somebody would need an emergency contraceptive for it can be even more shaming. Like why didn't you use this. Why didn't you use that. How come you're not using the pill. How come you're not using a condom. (R17, patient)

I think it's a problem people encounter while getting it. So the outcome of having the medication is still the same. But people feel bad about it after because of attitudes that they met from ... filling the prescription. (R23, patient)

\section{Prescribers and pharmacists}

First of all if you're sexually active it [EC] shouldn't be your main source of contraception. But obviously accidents do happen, and when those happen, then that should be an option for you and I will be willing to provide you that care. (R51, pharmacist)

I think that it should be sort of a last-ditch thing ... it shouldn't be used all the time ... I mean, normally I'm totally happy to dispense it. I don't want there to be unwanted pregnancies, unwanted abortions, unwanted children. But I sometimes feel like there should be a little bit more preparation done. (R52, pharmacist)

\section{Prescription status as a barrier}

If I had 2 options, one was a prescribed pill and one was an over-the-counter pill, I would go for the over-thecounter pill. Because I don't want to book an appointment with my doctor to go get the prescription, to then go to the pharmacy to pick up the medication. I just want to go directly to the pharmacy and get the pill. (R19, patient)

\section{Counter status}

"[We place it] behind the counter, so we want to make sure we don't miss anything. So even if someone come[s] to the counter request[ing] emergency contraception, the pharmacist will automatically provide counselling to the patient ... We dispense medication. And we have to provide counselling of everything that leaves the drugstore. Every kind of medication we provide counselling. And the counselling is done by the pharmacist. (R111, pharmacist)

Tension between pharmacist's duty to care and patient's desire for privacy and minimal contact

Someone might be really embarrassed to be in the pharmacy ... I know it's my experience of feeling embarrassed to be inside the pharmacy and want to get out as quickly as possible. Don't want to look at the pharmacist. You just want to get your pill and leave. (R16, patient)

\section{Medication stock and demand}

But for sure if the government allows ulipristal to be also prescribed by pharmacists then I think in general pharmacy is - it's a business. So they would do whatever that would make them money. So if they think that increasing the awareness of this new birth control pill that's effective for 5 days after rather than 3 days after, and more girls are aware of it and they kept - more girls are asking for it, then they would see it as a business opportunity. Then that would be what would make them, like, advertise or - to advocate for the change. (R31, pharmacist)

\section{Cost}

I think cost being the big one. (R17, patient). 
The critical opinions of some prescriber and pharmacist participants and the perceived shame of some patient participants indicated mutual discomfort related to accessing emergency contraception. Patient participants expressed feelings of shame for needing emergency contraception. Patients' lived experience and health care providers' beliefs about the acceptability of emergency contraceptive use can contribute to reinforcing patients' perceived shame beliefs.

\section{Theme 3: Health care system barriers (reinforcement)}

We identified multiple health system barriers for patients seeking emergency contraception, including prescription status, "behind-the-counter" access, limited supply due to perceptions of low demand, and cost. These barriers mapped to the theoretical domain of reinforcement, encouraging a behaviour through positive or negative stimulus.

Patients identified the prescription status of ulipristal acetate as a barrier, because they needed to take time away from work, find transportation or wait during unpredictable walkin clinic hours.

We also identified "counter status" (where emergency contraception is physically located) as a barrier. Although levonorgestrel is available "over the counter," pharmacists often keep it "behind the counter" to reinforce medication safety and patient counselling. From the patient's perspective, this practice inadvertently reinforced barriers to access and did not consider patient-identified characteristics of "safety," specifically privacy, mental wellness, and avoidance of shame or stigma.

Pharmacists' duty to counsel was in tension with patients' reported desire for privacy and minimal contact to mitigate shame and stigma. Many pharmacists reported that their pharmacies did not regularly stock ulipristal acetate because of perceived low demand and concerns about the costs of expired product to their business.

All participants cited cost as a barrier to emergency contraceptive access.

Multiple health system barriers affected access to emergency contraception and patients' help-seeking behaviours. Although ulipristal acetate is more effective than levonorgestrel in preventing pregnancy, participants identified multiple barriers to accessing this drug.

\section{Interpretation}

Since ulipristal acetate emergency contraception became available in $\mathrm{BC}$, the number of units sold and dispensed increased, while sales of levonorgestrel remained stable. Our quantitative analysis indicated that use of ulipristal acetate in Canada is low compared with levonorgestrel emergency contraception use. Qualitative data analysis identified potential reasons for this trend: low awareness about ulipristal acetate, shame and stigma, and health care system barriers, including prescription status and cost.

All participants identified low awareness of ulipristal acetate, consistent with previous studies. ${ }^{19,21}$ Similarly, Australian and European studies found that ulipristal acetate was not frequently supplied or sold because of a lack of clinical knowledge and new market status..$^{14,26}$ Our findings suggest a need to increase awareness and knowledge to optimize clinically recommended use of ulipristal acetate.

Our analyses illustrate that patients experienced stigma and shame in accessing emergency contraception, such that a patient may avoid pharmacy counselling with subsequent mistimed use of emergency contraception. Patients' experiences may have been in reaction to health care professionals' perception of emergency contraception as a last resort for preventing pregnancy and use of emergency contraception only if they "really screwed up." To facilitate implementation of ulipristal acetate in routine contraceptive care, it is critical to address beliefs about shame and stigma. Shame and stigma have been identified as determinants of health outcomes and health inequities, particularly in reproductive health. People who experience stigma have poorer health outcomes because of adjusted help-seeking behaviour. ${ }^{42}$

We identified multiple other barriers in patients' access to emergency contraception consistent with previous secret shopper studies. ${ }^{16-18}$ Our results offer a refined understanding of the "counter status" barrier - the desire to avoid shaming and limit social contact were salient to patients while pharmacists held concerns about their duty to provide safe medication counselling. The cost of medication is a known barrier. Prior research has shown that people from affluent areas were significantly more likely to use emergency contraception to prevent pregnancy than individuals from less affluent areas. ${ }^{43}$ Our results further identified that pharmacists were concerned about the cost to their business of expiry of stock in the event of low consumer demand.

Quantitatively, ulipristal acetate use is less frequent than use of levonorgestrel, which indicates the need for additional strategies to support expanded implementation. Despite current guidance from the Society of Obstetricians and Gynaecologists of Canada, ${ }^{1}$ levonorgestrel continues to be sold more frequently than ulipristal acetate. Qualitative data suggest knowledge, shame and stigma, and health system barriers (prescription status, cost) as opportunities to improve use of and access to emergency contraception. The complementary qualitative findings illuminate potential factors to explain less frequent use of ulipristal acetate: knowledge and multiple barriers both indicated low usage patterns, suggesting a need for additional strategies to support implementation.

\section{Limitations}

The strengths of this study included the use of populationbased data for dispensing of ulipristal acetate prescriptions and the multidisciplinary research advisory team, which provided diverse clinical and professional expertise.

The study findings must be interpreted in the context of several limitations. Our quantitative data sources did not capture inpatient prescriptions. In addition, sales and prescription data served as a proxy for medication use, but did not 
guarantee that individuals used the medications they obtained. We lacked provincial BMI data, which prevented analysis of emergency contraception method according to BMI category. For the qualitative data collection, there was no pilot-testing of the interview questions, nor was there any participant checking.

Our data reflect the use of ulipristal acetate in a single province of Canada, BC. Nevertheless, the barriers related to the prescription status of this drug are likely applicable to the rest of Canada because of the similarities among provincial health systems, the date of Health Canada authorization for ulipristal acetate and prescription coverage across the provinces.

\section{Conclusion}

In this study of ulipristal acetate use in BC, we found increased use of this agent over the study period, but we also identified multiple barriers to access. The data highlight factors potentially contributing to the low use of ulipristal acetate, including lack of knowledge on the part of health care professionals and patients, as well as multiple health care system barriers. Although these barriers may not be comprehensive or causal, our qualitative findings suggest reasons for the quantitative findings.

Opportunities for knowledge translation to improve access to emergency contraception include continuing medical education for providers and pharmacists about emergency contraception, curricular training for health care professionals to address issues of shame and stigma, education of the public about emergency contraception, and advocacy for health policy initiatives for subsidized, nonprescription (overthe-counter) emergency contraception.

\section{References}

1. Chapter 3. Emergency contraception. F Obstet Gynaecol Can 2015;37 (Suppl):S20-8.

2. Trussell J, Raymond EG, Cleland K. Emergency contraception: a last chance to prevent unintended pregnancy. Contemp Readings Law Soc 7ustice 2014. Available: https://heinonline.org/HOL/Page?handle=hein.journals/conreadlsj6\&id= $813 \&$ div $=100 \&$ collection $=$ journals $($ accessed 2020 Apr. 22). Login required to access content.

3. Sabourin JN, Burnett M. A review of therapeutic abortions and related areas of concern in Canada. 7 Obstet Gynaecol Can 2012;34:532-42.

4. Shen J, Che Y, Showell E, et al. Interventions for emergency contraception. Cochrane Database Syst Rev 2019;(1):CD001324.

5. Glasier AF, Cameron ST, Fine PM, et al. Ulipristal acetate versus levonorgestrel for emergency contraception: a randomised non-inferiority trial and meta-analysis. Lancet 2010;375:555-62.

6. Table 13-10-0096-20: Body mass index, overweight or obese, self-reported, adult, age groups (18 years and older). Ottawa: Statistics Canada. Available: https://www150.statcan.gc.ca/t1/tbl1/en/tv.action?pid=1310009620 (accessed 2020 Apr. 22).

7. Wong K, Hum S, McCarthy L, et al. Beyond Plan B: a qualitative study of Canadian pharmacists' emergency contraception counselling practices. $\mathcal{F}$ Obstet Gynaecol Can 2017;39:1021-7.

8. Leung VWY, Soon JA, Lynd LD, et al. Population-based evaluation of the effectiveness of two regimens for emergency contraception. Int 7 Gynaecol Obstet 2016;133:342-6.

9. Shoveller J, Chabot C, Soon JA, et al. Identifying barriers to emergency contraception use among young women from various sociocultural groups in British Columbia, Canada. Perspect Sex Reprod Health 2007;39:13-20.

10. Soon JA, Levine M, Ensom MHH, et al. The developing role of pharmacists in patient access to emergency contraception. Dis Manag Health Outcomes 2002;10:601-11.

11. Soon JA, Levine M, Osmond BL, et al. Effects of making emergency contraception available without a physician's prescription: a population-based study. CMAJ 2005;172:878-83.
12. Vanya M, Matuz M, Benko R, et al. Knowledge and opinion of pharmacists on emergency contraceptive pills in Hungary. Int 7 Clin Pharm 2017;39:594-600.

13. Milosavljevic J, Bogavac-Stanojevic N, Krajnovic D, et al. Serbian gynecologists' and pharmacists' beliefs about emergency contraception. Women Health 2017;57:508-19.

14. Collins JC, Schneider CR, Moles RJ. Emergency contraception supply in Australian pharmacies after the introduction of ulipristal acetate: a mystery shopping mixed-methods study. Contraception 2018;98:243-6.

15. Tavares MP, Foster AM. Emergency contraception in a public health emergency: exploring pharmacy availability in Brazil. Contraception 2016;94:109-14.

16. Bullock H, Steele S, Kurata N, et al. Pharmacy access to ulipristal acetate in Hawaii: Is a prescription enough? Contraception 2016;93:452-4.

17. Hussainy SY, Stewart K, Pham MP. A mystery caller evaluation of emergency contraception supply practices in community pharmacies in Victoria, Australia. Aust 7 Prim Health 2015;21:310-6.

18. Shigesato M, Elia J, Tschann M, et al. Pharmacy access to ulipristal acetate in major cities throughout the United States. Contraception 2018;97:264-9.

19. Milosavljevic J, Krajnovic D, Bogavac-Stanojevic N. Predictors of pharmacists' provision of emergency contraceptive pills. Health Care Women Int 2016;37: 1170-81.

20. Kiechle $M$, Neuenfeldt $M$. Experience with oral emergency contraception since the OTC switch in Germany. Arch Gynecol Obstet 2017;295:651-60.

21. Garrett KP, Widman L, Francis DB, et al. Emergency contraception: sources of information and perceptions of access among young adults. Women Health 2016;56:668-79.

22. Dawson A, Tran NT, Westley E, et al. Improving access to emergency contraception pills through strengthening service delivery and demand generation: a systematic review of current evidence in low and middle-income countries. PLoS One 2014;9:e109315.

23. Batur P, Cleland K, McNamara M, et al. Emergency contraception: a multispecialty survey of clinician knowledge and practices. Contraception 2016;93:145-52.

24. Bastianelli C, Rosato E, Farris M, et al. Emergency contraception: a survey of 1773 women. Eur 7 Contracept Reprod Health Care 2016;21:455-61.

25. Krassovics M, Virágh G. Usage patterns and attitudes towards emergency contraception: the International Emergency Contraception Research Initiative. Eur 7 Contracept Reprod Health Care 2016;21:310-7.

26. Italia S, Brand H. Status of emergency contraceptives in Europe one year after the European Medicines Agency's recommendation to switch ulipristal acetate to non-prescription status. Public Health Genomics 2016;19:203-10.

27. Fetters MD, Curry LA, Creswell JW. Achieving integration in mixed methods designs - principles and practices. Health Serv Res 2013;48:2134-56.

28. Census metropolitan area (CMA) and census agglomeration (CA). Ottawa: Statistics Canada; modified 2018 Sept. 17. Available: https://www150.statcan.gc.ca/n1/ pub/92-195-x/2011001/geo/cma-rmr/cma-rmr-eng.htm (accessed 2020 Apr. 24).

29. BC Ministry of Health [creator] (2011): PharmaNet. V2. BC Ministry of Health [publisher]. Data Extract. Data Stewardship Committee (2011). Available: http://www.popdata.bc.ca/data (accessed 2021 Nov. 1).

30. Dataset inventory. Vancouver: Health Data Research Network Canada; 2020. Available: https://www.hdrn.ca/en/inventory/ (accessed 2021 May 6).

31. 2019 ACTS annual report: statistical quality assurance applied to IQVIA's information offerings. Durham (NC): IVQIA; 2020. Available: https://www.iqvia.com/-/ media/iqvia/pdfs/library/publications/2019-acts-annual-report.pdf (accessed 2021 Apr. 4).

32. Collier R. Person-first language: what it means to be a "person". CMAf 2012;184:E935-6.

33. Atkins L, Francis J, Islam R, et al. A guide to using the Theoretical Domains Framework of behaviour change to investigate implementation problems. Implement Sci 2017;12:77.

34. Guetterman TC. Descriptions of sampling practices within five approaches to qualitative research in education and the health sciences. Forum Qual Soc Res 2015;16:25.

35. Palinkas LA, Horwitz SM, Green CA, et al. Purposeful sampling for qualitative data collection and analysis in mixed method implementation research. Adm Policy Ment Health 2015;42:533-44.

36. Hussainy SY, Ghosh A, Taft A, et al. Protocol for ACCESS: a qualitative study exploring barriers and facilitators to accessing the emergency contraceptive pill from community pharmacies in Australia. BM7 Open 2015;5:e10009.

37. Guest G, Bunce A, Johnson L. How many interviews are enough? An experiment with data saturation and variability. Field Methods 2006;18:59-82.

38. Saunders B, Sim J, Kingstone T, et al. Saturation in qualitative research: exploring its conceptualization and operationalization. Qual Quant 2018;52:1893-907.

39. Cane J, O'Connor D, Michie S. Validation of the theoretical domains framework for use in behaviour change and implementation research. Implement Sci 2012;7:37.

40. Ross L, Solinger R. Reproductive justice: an introduction. Berkeley (CA): University of California Press; 2017:1-351.

41. Reinharz S, Davidman L. Feminist methods in social research. Oxford (UK): Oxford University Press; 1992.

42. Harris LH. Stigma and abortion complications in the United States. Obstet Gynecol 2012;120:1472-4.

43. Cameron ST, Gordon R, Glasier A. The effect on use of making emergency contraception available free of charge. Contraception 2012;86:366-9. 
Affiliations: Department of Obstetrics and Gynaecology (Chan, Munro, Fitzsimmons, Renner), Centre for Health Evaluation and Outcome Sciences (Munro), Department of Family Practice (Schummers, Soon) and Faculty of Pharmaceutical Sciences (Soon, Ragsdale), University of British Columbia; Women's Health Research Institute (Albert, Mackenzie), British Columbia Women's Hospital, Vancouver, BC

Contributors: Michelle Chan and Regina Renner designed and developed the study concept, with input from all coauthors. Sarah Munro and Brian Fitzsimmons substantially contributed to the design of qualitative methods. Laura Schummers, Arianne Albert and Brian Fitzsimmons substantially contributed to the design of quantitative methods. Judith Soon and Parkash Ragsdale contributed advice for quantitative sales data and leveraged pharmaceutical networks for recruitment. Frannie Mackenzie substantially contributed to participant recruitment and data management. Michelle Chan collected qualitative and quantitative data, and performed analysis of the qualitative data. Arianne Albert performed management and analysis of the quantitative data. Sarah Munro substantially assisted with analysis of the qualitative data. All of the authors substantially contributed to the interpretation of the study results. Michelle Chan presented a review of the integrated analysis, to which all authors contributed. Michelle Chan wrote the first draft of the manuscript and led the manuscript revisions. All of the authors contributed to manuscript revisions, reviewed and approved the final manuscript for publication, and agreed to be accountable for the work.

Funding: This study was funded by the Society of Family Planning Research Fund (SFPRF 19-03). Laura Schummers was supported by a trainee award from the Michael Smith Foundation for Health Research
(17934) and a Postdoctoral Health System Impact Fellowship jointly funded by the Canadian Institutes of Health Research (H17-160383) and the British Columbia Ministry of Health (1818). Sarah Munro was supported by a Michael Smith Foundation for Health Research Scholar Award (18270).

Content licence: This is an Open Access article distributed in accordance with the terms of the Creative Commons Attribution (CC BY-NC-ND 4.0) licence, which permits use, distribution and reproduction in any medium, provided that the original publication is properly cited, the use is noncommercial (i.e., research or educational use), and no modifications or adaptations are made. See: https://creativecommons.org/licenses/by-nc-nd/4.0/

Data sharing: The PharmaNet data are available for use by other researchers; access is regulated and controlled by Population Data BC. IQVIA data are available for use by other researchers; access is regulated and controlled by IQVIA. The qualitative data are not available for use by other researchers.

Disclaimer: All inferences, opinions, and conclusions drawn in this study are those of the authors, and do not reflect the opinions or policies of the data steward(s) or PharmaNet (BC Ministry of Health).

Acknowledgements: The authors gratefully acknowledge research and resource support from Nicole Prestley of the Women's Health Research Institute.

Supplemental information: For reviewer comments and the original submission of this manuscript, please see www.cmajopen.ca/content/9/4/ E1097/suppl/DC1 